\title{
Conduction Band Engineering of Half-Heusler Thermoelectrics Using Orbital Chemistry
}

Shuping Guo, ${ }^{\dagger, \ddagger}$ Shashwat Anand, ${ }^{\ddagger}$ Madison K. Brod, ${ }^{\ddagger}$ Yongsheng Zhang, ${ }^{*, \dagger, \S}$

and G. Jeffrey Snyder*,

$\dagger$ Key Laboratory of Materials Physics, Institute of Solid State Physics, Chinese Academy of Sciences, Hefei 230031, China.

$\ddagger$ Department of Materials Science and Engineering, Northwestern University, Evanston, IL 60208, USA.

【Institute for Theoretical Solid State Physics, Leibniz IFW Dresden, 01069 Dresden, Germany

$\S$ Science Island Branch of Graduate School, University of Science and Technology of China, Hefei 230026, China.

E-mail: yshzhang@theory.issp.ac.cn; jeff.snyder@northwestern.edu

\begin{abstract}
Semiconducting half-Heusler $(\mathrm{HH}, X Y Z)$ phases are promising thermoelectric materials owing to their versatile electronic properties. Because the valence band of half-Heusler phases benefits from the valence band extrema at several high-symmetry points in the Brillouin zone (BZ), it is possible to engineer better p-type HH materials through band convergence. However, the thermoelectric studies of n-type HH phases have been lagging behind since the conduction band minimum is always at the same high-symmetry point $(\mathrm{X})$ in the BZ, giving the impression that there is little opportunity for band engineering. Here we study the n-type orbital phase diagram of $69 \mathrm{HHs}$,
\end{abstract}


and show that there are two competing conduction bands with very different effective masses actually at the same X point in the BZ, which can be engineered to be converged. The two conduction bands are dominated by the $d$ orbitals of $X$ and $Y$ atoms, respectively. The energy offset between the two bands depends on the difference in electron configuration and electronegativity of the $X$ and $Y$ atoms. Based on the orbital phase diagram, we provide the strategy to engineer the conduction band convergence by mixing the $\mathrm{HH}$ compounds with the reverse band offsets. We demonstrate the strategy by alloying VCoSn and TaCoSn. The $\mathrm{V}_{0.5} \mathrm{Ta}_{0.5} \mathrm{CoSn}$ mixture presents the high conduction band convergence and corresponding significantly larger density-of-states effective mass than either VCoSn or TaCoSn. Our work indicates that analyzing the orbital character of band edges provides new insight into engineering thermoelectric performance of $\mathrm{HH}$ compounds.

\section{Introduction}

Half-Heusler $(\mathrm{HH})$ compounds are promising functional materials, e.g. thermoelectrics, ${ }^{1}$ optoelectronics, ${ }^{2}$ spintronics ${ }^{3}$ and topological insulators, ${ }^{4}$ because of their versatile and complex electronic properties, as well as good thermal and mechanical stability. ${ }^{5}$ As shown in Fig. 1, the crystal structure of $\mathrm{HH}$ is composed of four face-centered-cubic (FCC) sublattices, with three of them occupied by $X, Y$ and $Z$ elements. When the constituent elements are valence balanced, ${ }^{6,7}$ the compounds generally exhibit semiconducting properties. Therefore, valence balanced $\mathrm{HH}$ family has long been used for practical high-temperature thermoelectric applications. $^{8}$

The thermoelectric performance is characterized by the figure of merit, $z T, z T=S^{2} \sigma T /\left(\kappa_{e}+\right.$ $\kappa_{l}$ ), where $S$ is the Seebeck coefficient, $\sigma$ the electrical conductivity, $\kappa_{e}$ the electronic thermal conductivity and $\kappa_{l}$ the lattice contribution to the thermal conductivity. But the Seebeck coefficient and electrical conductivity are inversely coupled by carrier concentration. ${ }^{1}$ Thus, improving Seebeck coefficient and electrical conductivity at the same time is a long- 
standing challenge. The electronic properties are primarily determined by weighted mobility, ${ }^{9} \mu\left(m_{d}^{*} / m_{e}\right)^{3 / 2}$, where large $m_{d}^{*}$ (density-of-states effective mass) is favorable for large Seebeck coefficient and high $\mu$ (charge carrier mobility) is essential for high electrical conductivity. Large band degeneracy can boost $m_{d}^{* 10,11}$ without changing carrier mobility, leading to high weighted mobility ${ }^{9}$ and then high thermoelectric performance. ${ }^{12}$ As a consequence, modifying the relative energy of band edges (band engineering) has been used extensively as an effective strategy to improve the performance of thermoelectric materials ${ }^{13}$ where band degeneracy $\left(N_{b}\right)$ can be seen as a simple and effective descriptor for good electronic properties for thermoelectric applications.

There has been great interest in engineering the valence bands and improve the thermoelectric performance of $\mathrm{HH}$ compounds. As a result, the $z T$ of p-type NbFeSb-based ${ }^{14} \mathrm{HH}$ is reported to be 1.5 at high temperature. These p-type HHs have valence bands with high valley degeneracy (e.g $N_{v}^{L}=4, N_{v}^{W}=6$ ) which contributes to its high power factor. ${ }^{15,16}$ Consequently, several promising p-type $\mathrm{HH}$ candidates are found with large valence band degeneracy, like $N_{b}^{L}=8$ (with two orbital degeneracy) for NbFeSb and TaFeSb ${ }^{17}$ and $N_{b}^{L+\Gamma}$ $=10$ (not including the spin-orbit split off band at $\Gamma$ ) for $\mathrm{ZrCoBi}^{18}$ leading to $z T \mathrm{~s}$ of 1.5 and 1.4, respectively. In contrast, there are few promising $\mathrm{n}$-type $\mathrm{HH}$ thermoelectric candidates reported. The best n-type $\mathrm{HH}$ remains $\mathrm{ZrNiSn-based}\left(N_{v}^{X}=3\right)$ with $z T$ values up to about 1.5 at $700 \mathrm{~K} .{ }^{19}$ All the works of ZrNiSn focus on reduction of lattice conductivity from 7 to $3 \mathrm{Wm}^{-1} \mathrm{~K}^{-1}$ at $300 \mathrm{~K} .{ }^{20,21}$ Increasing the band degeneracy through conduction band convergence which has been long-time ignored might be a good way to further improve the thermoelectric performance of n-type $\mathrm{HH}$ compounds.

Engineering $\mathrm{HH}$ thermoelectric materials based on structural chemistry is an effective way to understand the underlying mechanisms and propose feasible guidelines for improvements. ${ }^{22}$ For instance, identifying the atomic orbital contribution to the valence and conduction band edges, which mainly determine the electronic transport, can guide band engineering. Recently, Dylla et al. ${ }^{23}$ studied the orbital constitutions of the valence band edges 
(presenting an orbital contribution diagram). Over 50 known HH compounds were sorted into three groupings distinguished by the location of the valence band edge (at $\Gamma, \mathrm{L}$ or $\mathrm{W}$ ): the band extremum is located at $\Gamma$ or $\mathrm{L}$ depending on which atom's $d$-orbitals $(X$ or $Y$ in $X Y Z$ Half Heusler) dominates the valence band edge, the band extremum can also be at $W$ with high valley degeneracy $\left(N_{v}^{W}=6\right)$ when the $Z$ atom's $p$-orbitals further contribute to the valence band edge. The chemical trends give valuable guidelines to engineer band convergence as well as high valley degeneracy. Unlike the valence band, the conduction band of almost all $\mathrm{HH}$ thermoelectrics is invariably a single band located at the $\mathrm{X}$ point in the Brillouin zone which makes it appear like there is little opportunity for band engineering and may explain the lack of effort to study the conduction band structure.

In this work, we examine the conduction band edges in $69 \mathrm{HH}$ semiconductors and find that there are actually two different conduction bands at $\mathrm{X}$ point, one dominated by the $d$-orbitals of the $X$ atom and the other dominated by the $d$-orbitals of the $Y$ atom. This casts new insight into engineering band convergence in n-type thermoelectric $\mathrm{HH}$ compounds, which is successfully demonstrated in the mixture of VCoSn and TaCoSn.

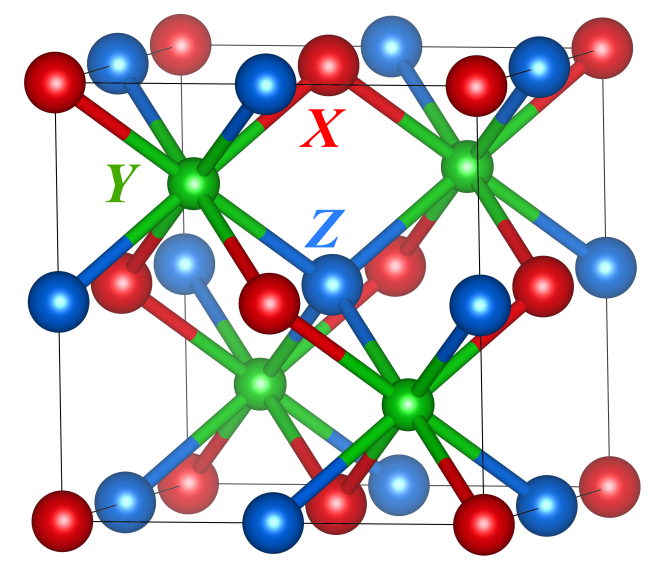

Figure 1: Crystal structure of the half-Heusler compound. The red, green and blue spheres represent the $X, Y$ and $Z$ atoms, respectively. 


\section{Methodology}

\section{Density-Functional Theory}

We use the projected augmented wave $(\mathrm{PAW})^{24}$ scheme with the generalized gradient approximation of Perdew-Burke-Ernzerhof $(\mathrm{PBE})^{25}$ electronic exchange correlation functional in the Vienna ab initio simulation package (VASP). The energy cutoff is $1.3 E_{\max } \mathrm{eV}$, where $E_{\max }$ is the largest energy of the default maximum energy cutoffs of $X, Y$ and $Z$ atoms. We use the Monkhorst-Pack ${ }^{26}$ approach to sample the Brillouin zones with a roughly constant density of k-points $\left(30 \AA^{3}\right)$ for structure relaxation, and a highly dense density $\left(60 \AA^{3}\right)$ for electronic structure calculation. The conductivity effective mass $\left(m_{c}^{*}\right)$ is calculated by Boltztrap $2^{27}$ code for electrical conductivity using the constant relaxation time approximation (CRTA, $\tau=10 \mathrm{fs}$ ). ${ }^{28}$

$$
\left(m_{c \alpha \beta}^{*}(T ; \mu)\right)^{-1}=\frac{\sigma_{\alpha \beta(T ; \mu)}}{e^{2} \tau} \times \frac{1}{n(T ; \mu)}
$$

\section{Special Quasi-Random Structure (SQS) Method}

Solid solutions are typically synthesized to lower the thermal conductivity ${ }^{17,18}$ but can also affect the electronic properties. ${ }^{29}$ To describe the disordered state, large supercells are needed, leading to time-consuming computational cost. Special quasi-random structures (SQS) ${ }^{30}$ are chosen with close multisite correlation functions to the ideal disordered state in order to mimic the disorder of solid solution with a small supercell. SQS structures have been successfully used to characterize the properties of experimental solid solutions, like ternary Sr-Pb-S, ${ }^{31}$ binary BCC alloys ${ }^{32}$ as well as half-Heusler systems. ${ }^{11,33}$ Wolverton $^{34}$ et al. predicted SQS structure with 48 atoms is used to study the $\mathrm{V}$ and Ta mixing in one of the FCC sublattices for the $\mathrm{V}_{0.5} \mathrm{Ta}_{0.5} \mathrm{CoSn}$ solid solutions. To compare the band structures, BandUP ${ }^{35}$ is used to unfold the band structure of the SQS into the primitive Brillouin zone of the pristine compound. The density-of-states (DOS) effective mass $\left(m_{d}^{*}\right)$ of $\mathrm{SQS} \mathrm{V}_{0.5} \mathrm{Ta}_{0.5} \mathrm{CoSn}$ is 
calculated by fitting the DOS around the Fermi level.

$$
\operatorname{DOS}(E)=\frac{1}{2 \pi^{2}}\left(\frac{2 m_{d}^{*}}{\hbar^{2}}\right)^{3 / 2} E^{1 / 2}
$$

\section{Results and Discussion}

\section{Orbital Diagram of Conduction Band Edges}

Electronic structures provide important information relating to electronic transport properties. Band convergence leads to high DOS that increases the electronic quality factor ${ }^{36}$ (or weighted mobility ${ }^{9}$ ) as long as there is not a proportional decrease in mobility, e.g. from intervalley scattering. ${ }^{37}$ Engineering the band edges to achieve high band degeneracy is therefore a clear strategy to improve thermoelectric performance. A simple model is needed to engineer the band convergence by doping or alloying. Since the electronic states correspond to combinations of atomic orbitals, variations in the fractional contribution from each orbital type to a band should influence the band shape as well as the energy of the band edge.

The valence band edge of Half-Heusler semiconductors can be at $\Gamma$, the highest symmetry point in the Brillouin zone with $N_{v}^{\Gamma}=1$, or at other points with higher valley degeneracy, like $N_{v}^{L}=4$ at the L point and $N_{v}^{W}=6$ at the $\mathrm{W}$ point. ${ }^{23}$ But the conduction band edge is usually located at X (or sometimes $\Gamma$ point), with valley degeneracy of $N_{v}^{X}=3$. For the HH family, although each phase has the same crystal structure, it has wide chemistry flexibility since there are many possible elements for each site. Different elements result in different orbital contributions, leading to various band structures in HH compounds. Therefore, mapping the change of conduction band structures with various compositions of $\mathrm{HH}$ is a powerful strategy to elucidate the primary sources of variance among the reciprocal-space electronic structures.

To quantitatively explore how chemical composition (atomic orbitals) affect electronic structures, we select 69 kinds of stable (computationally stable at $T=0 \mathrm{~K}$ ) $\mathrm{HH}$ compounds 
(a)

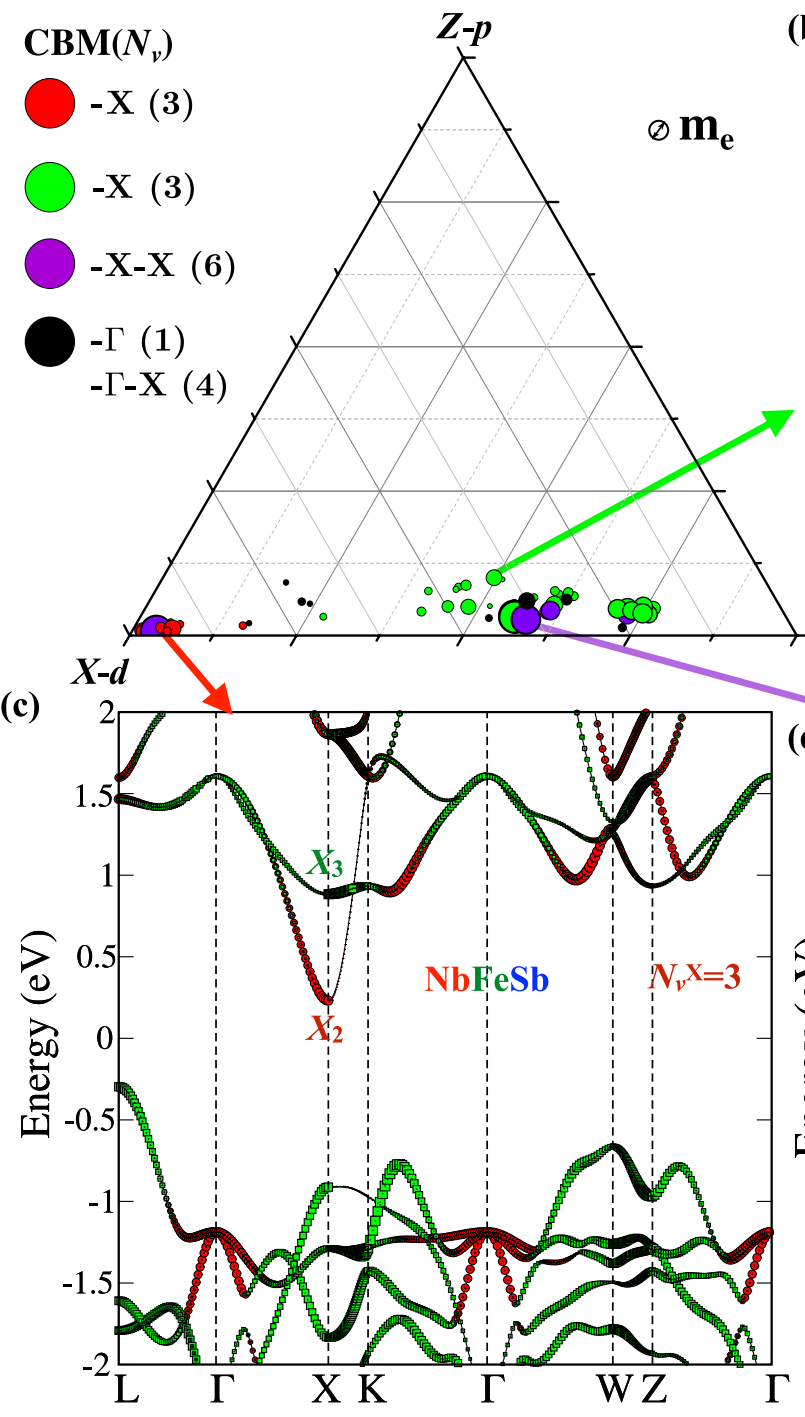

(b)
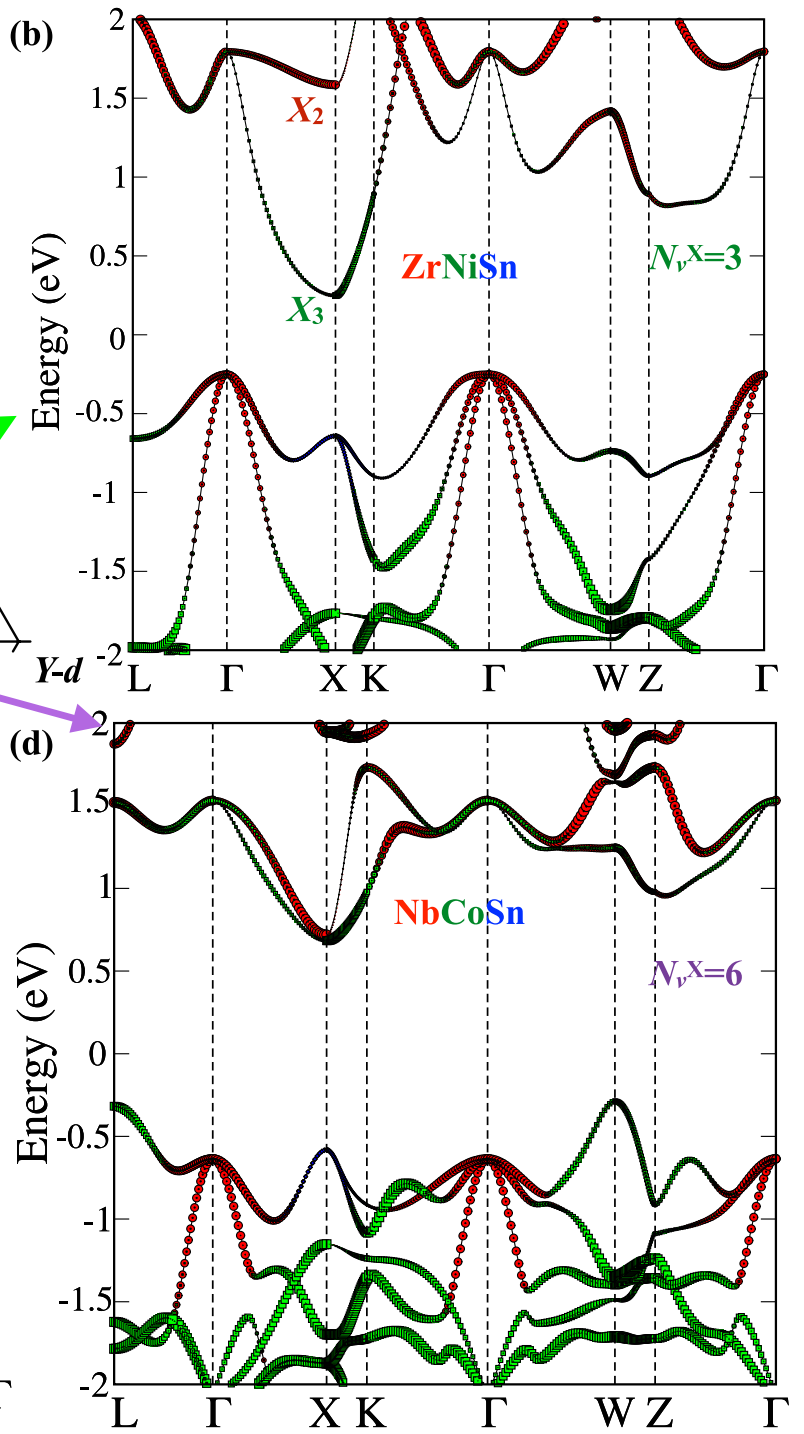

Figure 2: (a) The conduction band edges of half-Heusler compounds are primarily composed of the $d$ orbitals of the $X$ and $Y$ elements. The size of circles represents the n-type conductivity mass with the carrier concentration of $10^{20} \mathrm{~cm}^{-3}$ under $900 \mathrm{~K}$. Two different conduction band minima $(\mathrm{CBM})$ arise at the $\mathrm{X}$ point (valley degeneracy $N_{v}=3$ ) in the Brillouin zone, one dominated by the $d$-orbital of $X$ atoms (minimum at energy $E_{X_{2}}$, red sphere) and one dominated by the $d$-orbital of $Y$ atoms (minimum at energy $E_{X_{3}}$, green sphere). In some compounds, such as ZrNiSn (b) the $Y$ atom forms the CBM while in other compounds such as NbFeSb (c) the $X$ atom forms the CBM. These two conductions bands are effectively converged in NbCoSn (d) with the energy difference of the CBMs being below a few $k_{B} T$, doubling the effective valley degeneracy to $6\left(N_{b}=6\right)$. 
and project the density of states into the atomic orbitals, from which we can establish the conduction band orbital phase diagram. As shown in Fig. 2a, we can see that it is the contribution of $d$ orbitals of the $X$ and $Y$ elements that mainly distinguish the conduction band edges. More importantly, although most HH compounds have conduction band edge at the $\mathrm{X}$ point there are actually two classes of conduction band edges. The conduction band minima of the both bands locate at the X point in BZ (Fig. 2b and 2c), one primarily arising from the $d$ orbitals of $X$ (red circles, making conduction band minimum at $X_{2}$ ) and another from $Y$ atoms (green circles, making conduction band minimum at $X_{3}$ ). One representative of the second class is the well-known n-type HH ZrNiSn (Fig. 2b): the conduction band minimum (CBM) locates at X point coming from the $d$ orbital of Ni atom (green line, at $X_{3}$ ) and a secondary CBM at X point stemming from $d$ orbital of Zr atom (red line, at $X_{2}$ ) with the energy difference $\left(E_{X_{3}}-E_{X_{2}}\right)$ of $-1.34 \mathrm{eV}$. In contrast, one representative of first class is the good p-type $\mathrm{HH} \mathrm{NbFeSb}$ (Fig. 2c): the conduction band minimum is dominated by the Nb- $d$ orbital (red line, $X_{2}$ ) and a secondary CBM attributed by the Fe- $d$ orbital (green line, $\left.X_{3}\right)$, which are both laying on $\mathrm{X}$ point, with the energy offset $\left(E_{X_{3}}-E_{X_{2}}\right)$ of $0.65 \mathrm{eV}$.

When the energy offset of these two types of CBM at $\mathrm{X}$ point $\left(X_{3}\right.$ and $\left.X_{2}\right)$ is below a few $k_{B} T$ implying that both two bands contribute to carrier transport, e.g. NbCoSn (Fig. 2d): the energy difference between $X_{3}$ and $X_{2}$ is $0.04 \mathrm{eV}$. In this case, the band degeneracy reaches six. The effective mass of $X_{2}$ (red line) is noticeably lighter than the $X_{3}$ (green line), indicating the coexistence of light and heavy band edges. ZrRhBi is another compound with effectively converged CBMs (Fig. S9d). There is a flat band between CBM$X_{3}$ (contributed by $d$ orbital of $Y$ atom) and $\Gamma$ point $(\Delta E=0.03 \mathrm{eV})$, with the CBM- $X_{2}$ (attributed by $d$ orbital of $X$ atom) close in energy $(\Delta E=0.25 \mathrm{eV}$ ), which should have a complex Fermi surface with high band degeneracy. ${ }^{38,39}$ The orbital diversity of conduction band edges indicates larger degeneracy can be achieved by engineering the energy offset. 


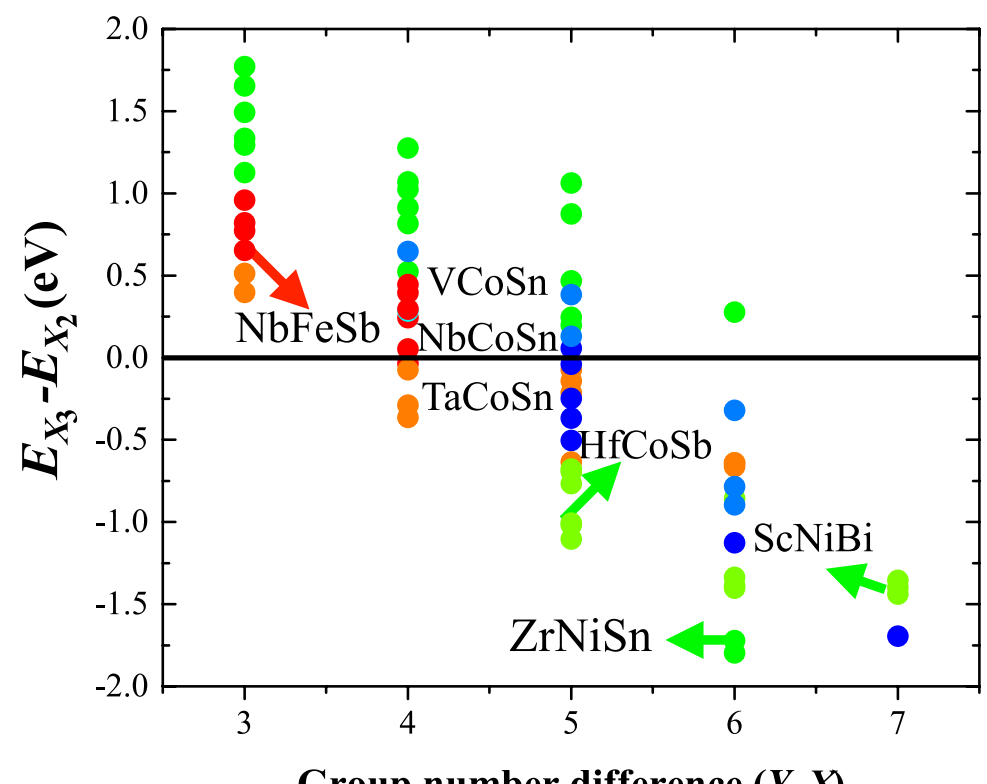

Group number difference $(\boldsymbol{Y}-\boldsymbol{X})$

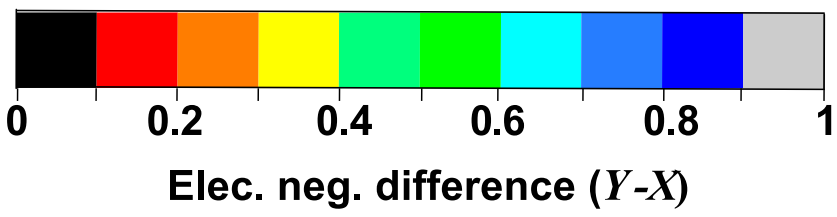

Figure 3: The difference in valence electron configuration (group number) and electronegativity of $X$ and $Y$ species determines the energy offset between the two conduction bands at $\mathrm{X}\left(E_{X_{3}}-E_{X_{2}}\right)$ point. 


\section{Descriptor for Band Convergence of N-Type HH}

Engineering the energy offset $\left(E_{X_{3}}-E_{X_{2}}\right)$ to realize band degeneracy can tune thermoelectric performance, which depends on adjusting the relative energies of the atomic orbitals that constitute the conduction bands. Atomic orbitals from $X, Y$ and $Z$ atoms have bonding and anti-bonding interactions, which lead to the formation of the bands. It is reported that the simplified crystal orbital diagram for a prototypical half-Heusler compound at X point, the competing CBMs are composed of either a non-bonding $d$-orbital states from the $X$ atom and anti-bonding state comprised of mostly $Y-d$ and $X-d$ orbitals. ${ }^{40}$ The chemical difference between $X$ and $Y$ atoms influences the relative energy difference between the atomic orbitals, and hence, the relative position of two distinct CBM at X point.

Fig. 3 shows the relationships between energy offset of two kinds of $\mathrm{X}$ points with valence electron configuration difference and electronegativity difference (color bar). The energy offset of $E_{X_{3}}$ and $E_{X_{2}}$ shows a linear relationship to the group number difference (the valance difference). Clearly, the valence electron difference, or the group or column number difference on the periodic table is a good descriptor for the energy difference. The variation in the relative energies of the two competing CBMs modified by group number difference between $X$ and $Y$ atoms is due to the increase in the on-site energy of the atomic orbitals moving across the row on the Periodic Table (left to right). ${ }^{41}$ Therefore, the compounds with a small valence difference, like $\mathrm{NbFeSb}$, have the conduction band minimum (at X point with energy $E_{X_{2}}$ ) dominated by the $d$ orbital of the $X$ element. In contrast, compounds with large valence differences, such as HfCoSb, have the conduction band minimum (at X point with energy $E_{X_{3}}$ ) dominated by the $d$ orbital of the $Y$ atom. In this case, double mixing at $X$ and $Y$ sites with opposite energy offset (like NbFeSb and $\mathrm{HfCoSb}$ ) can promote band convergence.

In addition, electronegativity, which characterizes the ionization energy of the element ${ }^{42}$ has the secondary, but important, influence on the energies of the atomic orbitals. Therefore, for the isovalent compounds (like VCoSn and TaCoSn), the second most important 


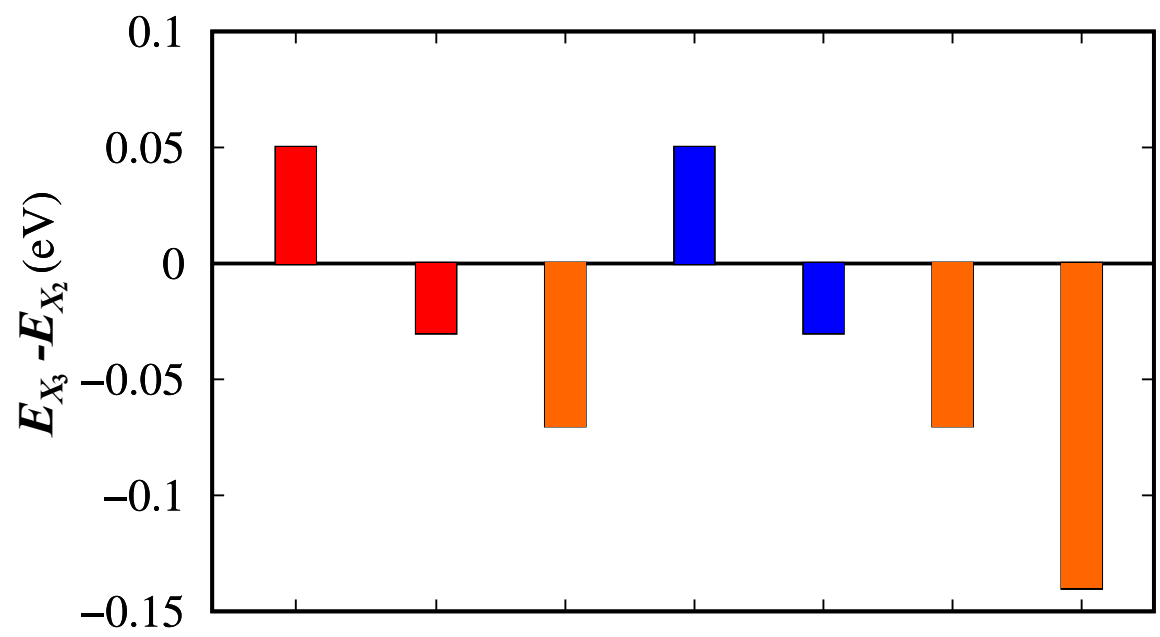

NbCoGe NbCoSn TaCoSi HfIrSb ZrRhAs TiCoAs TiCoSb

Figure 4: The energy difference of $E_{X_{3}}-E_{X_{2}}$ of seven compounds with small energy difference (near band convergence). The colors are the same as used in Figure 3, representing the electronegativity difference of $X$ and $Y$ species.

descriptor is the difference of electronegativity between the $X$ and $Y$ atoms. The systems with smaller electronegativity differences (red and yellow solid circles in Fig. 3), which imply more covalent bonding, tend to reduce the $E_{X_{3}}-E_{X_{2}}$ energy offset. The relationship between energy offset and these two descriptors can provide feasible guidance to engineer the band convergence. The energy offsets and projected band structures of all $69 \mathrm{HH}$ compounds are given in Table S1 and Fig. S1-9, respectively.

When the group number differences are four and five, there are several systems with the energy offset below a few $k_{B} T$, which should effectively have both conduction bands converged. As shown in Fig. 4, there are seven compounds (NbCoGe, NbCoSn, TaCoSi, HfIrSb, ZrRhAs, TiCoAs, TiCoSb) possess large band degeneracy of six with energy offset below a few $k_{B} T$. The colors are the same as used in Fig. 3, representing the difference of electronegativity. For other compounds with a large $E_{X_{3}}-E_{X_{2}}$ energy offset, mixing two $\mathrm{HH}$ compounds with an opposite energy offset could also lead to band convergence. 

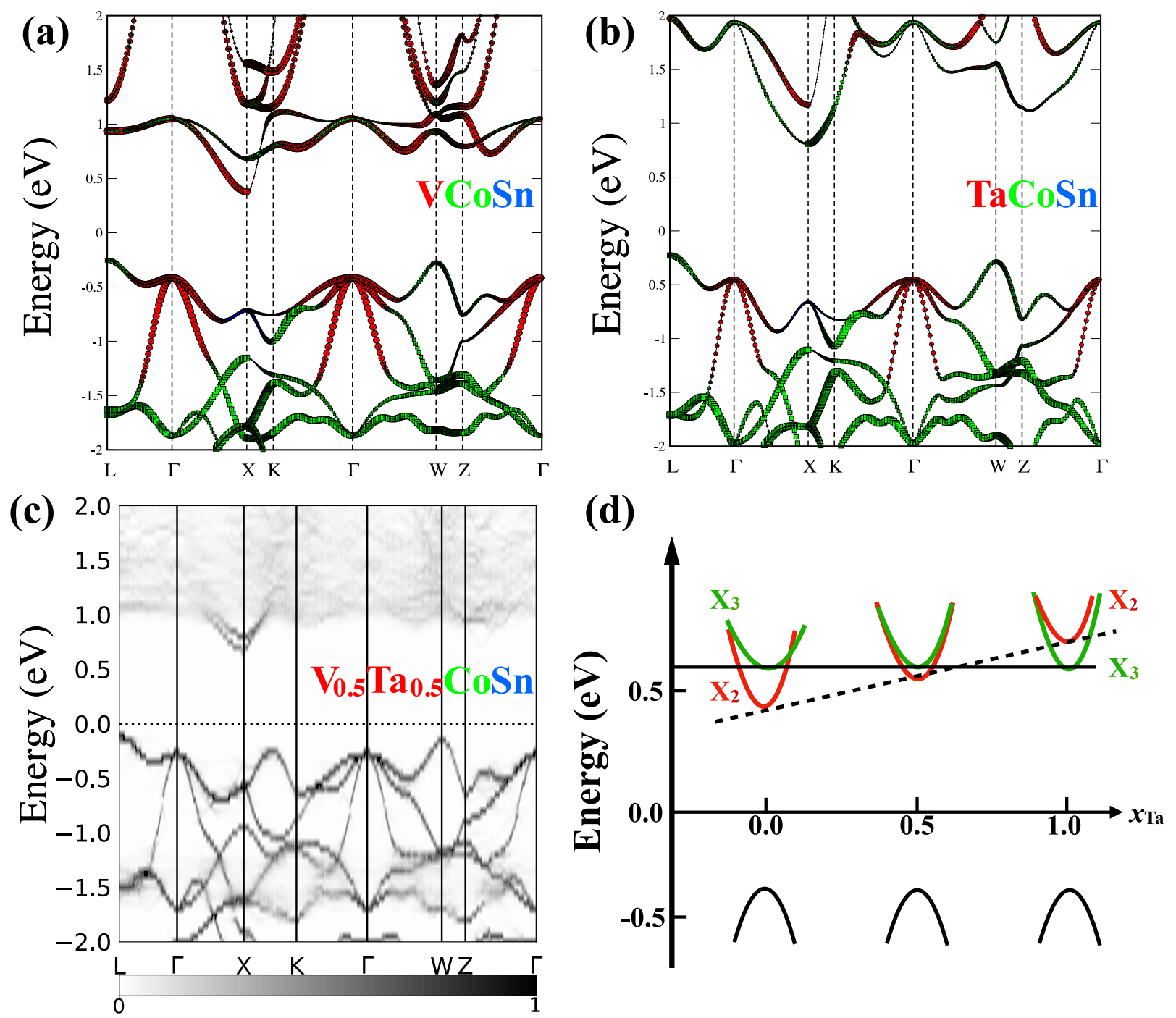

Figure 5: The projected band structures of (a) VCoSn and (b) TaCoSn. Unfolded band structure of (c) $\mathrm{V}_{0.5} \mathrm{Ta}_{0.5} \mathrm{CoSn}$ (SQS). The relative energy of the two conduction bands $\left(X_{2}\right.$ and $\left.X_{3}\right)(\mathrm{d})$ changes with the doping concentration of Ta in $\mathrm{V}_{1-x} \mathrm{Ta}_{x} \mathrm{CoSn}$ leading to band convergence near $x=0.5$. 


\section{Alloying to Achieve Conduction Band Convergence}

From the last section, we can see that mixing two isovalent compounds with opposite energy offset can realize band degeneracy by tuning electronegativity difference. To validate this, we choose two isovalent systems (VCoSn and TaCoSn) with reverse energy offset to see how the changing of electronegativity can engineer the $E_{X_{3}}-E_{X_{2}}$ energy offset. As shown in Fig. 5, the $E_{X_{3}}-E_{X_{2}}$ energy offsets for VCoSn and TaCoSn are 0.30 and $-0.36 \mathrm{eV}$, respectively. Based on a simple tight-bonding model, ${ }^{40,43}$ it can be shown that the on-site energy of an atomic orbital state affects the energy of the molecular orbital states which most closely resemble the atomic orbital state. That is, modifying the $X-d$ orbital has a greater influence on the energy of the non-bonding CBM $\left(E_{X_{2}}\right)$, while tuning the on-site energy of the $Y$-d atom has a greater influence on the anti-bonding $\mathrm{CBM}\left(E_{X_{3}}\right)$. Therefore, mixing Ta in VCoSn can increase the relative energy of $X_{2}$ then lead to the band convergence of $X_{2}$ and $X_{3}$. We choose SQS with the mixing concentrations of $50 \%$ Ta to study the electronic properties of solid solutions, then unfold its band structure to the primitive cell. For $\mathrm{V}_{0.5} \mathrm{Ta}_{0.5} \mathrm{CoSn}$ (Fig. 5c), the energy offset is around $0.15 \mathrm{eV}$, implying these two bands are converged with the degeneracy of six (Fig. 5d). It is clear that mixing $\mathrm{V}$ and Ta can modify the relative positions of $X_{2}$ and $X_{3}$. The convergence of light $\left(X_{2}\right)$ and heavy $\left(X_{3}\right)$ bands contributes to high DOS effective mass.

We then calculate the density-of-states of $\mathrm{V}_{0.5} \mathrm{Ta}_{0.5} \mathrm{CoSn}$, VCoSn and TaCoSn (Fig. 6). To check the DOS change with V-Ta mixing, we align their conduction band edges. It is clear that $\mathrm{V}_{0.5} \mathrm{Ta}_{0.5} \mathrm{CoSn}$ has larger DOS around CBM than VCoSn and TaCoSn. Correspondingly, the DOS effective mass of $\mathrm{V}_{0.5} \mathrm{Ta}_{0.5} \operatorname{CoSn}\left(3.49 m_{e}\right)$ is significantly higher than those of VCoSn $\left(1.9 m_{e}\right)$ and TaCoSn $\left(2.96 m_{e}\right)$. The high DOS effective mass of $\mathrm{V}_{0.5} \mathrm{Ta}_{0.5} \mathrm{CoSn}$ is obviously

contributed by the converged heavy and light bands, which is induced by the decreased $E_{X_{3}}{ }^{-}$ $E_{X_{2}}$ energy offset (Fig. 5c) in the mixture. Therefore, it would boost Seebeck coefficient without deteriorating the carrier mobility. Additionally, the introduced mass fluctuation (V and Ta) would scatter phonons and further suppress the lattice thermal conductivity as well. 


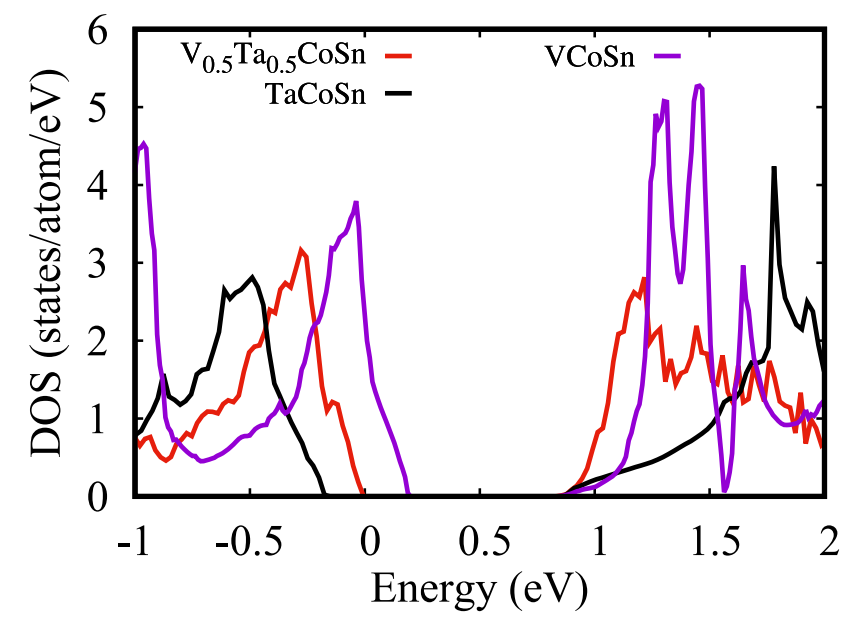

Figure 6: Density-of-states of SQS- $\mathrm{V}_{0.5} \mathrm{Ta}_{0.5} \mathrm{CoSn}$ (red line), VCoSn (purple line) and TaCoSn (black line).

Therefore, engineering the conduction band convergence based on our methodology would essentially improve the thermoelectric performance of n-type HH compounds.

\section{Conclusion}

We construct the n-type orbital phase diagram with 69 compositions of half-Heusler $X Y Z$ compounds and find that there is orbital diversity of conduction band edges. Two major classes of conduction band edges (both at the $\mathrm{X}$ point) are dominated by the $d$ orbitals of either the $X$ (with energy $E_{X_{2}}$ ) or $Y$ (with energy $E_{X_{3}}$ ) atom. The energy offset $E_{X_{3}}-E_{X_{2}}$ is determined by the valence (group number in the periodic table) and electronegativity difference between $X$ and $Y$ species to a lesser extent. Mixing two pristine compounds (VCoSn and TaCoSn) with opposite energy offset in 1:1 ratio can successfully converge $X_{3}$ and $X_{2}$ bands. As a result, SQS- $\mathrm{V}_{0.5} \mathrm{Ta}_{0.5} \mathrm{CoSn}$ exhibits a significantly higher n-type DOS effective mass than those of VCoSn and TaCoSn. Such an orbital diagram provides novel insight into understanding the underlying mechanisms behind the band energies and provides effective strategies to engineer high band degeneracy and better thermoelectric performance of half-Heusler compounds. 


\section{Acknowledgement}

Y. Z. acknowledges support from National Natural Science Foundation of China Grant No. 11774347. S. A., M. B. and G. J. S. acknowledge support by NSF-DMREF Award No. 1729487 and the U.S. Department of Energy, Office of Energy Efficiency and Renewable Energy (EERE) Award DE-AC02-76SF00515. S. G. acknowledges support from the China Scholarship Council (CSC) and technical assistance of Ulrike Nitzsche.

\section{Supporting Information Available}

All the energy offsets, valence differences, electronegativity differences of $X$ and $Y$ atoms, conductivity effective masses and projected band structures of $69 \mathrm{HH}$ compounds.

\section{References}

(1) Snyder, G. J.; Toberer, E. S. Complex thermoelectric materials. Nat. Mater. 2008, 7, $105-114$.

(2) Kieven, D.; Klenk, R.; Naghavi, S.; Felser, C.; Gruhn, T. I-II-V half-Heusler compounds for optoelectronics: Ab initio calculations. Phys. Rev. B 2010, 81, 075208.

(3) Ma, J.; Hegde, V. I.; Munira, K.; Xie, Y.; Keshavarz, S.; Mildebrath, D. T.; Wolverton, C.; Ghosh, A. W.; Butler, W. Computational investigation of half-Heusler compounds for spintronics applications. Phys. Rev. B 2017, 95, 024411.

(4) Lin, H.; Wray, L. A.; Xia, Y.; Xu, S.; Jia, S.; Cava, R. J.; Bansil, A.; Hasan, M. Z. Half-Heusler ternary compounds as new multifunctional experimental platforms for topological quantum phenomena. Nat. Mater. 2010, 9, 546-549.

(5) Al Malki, M.; Qiu, Q.; Zhu, T.; Snyder, G.; Dunand, D. Creep behavior and postcreep 
thermoelectric performance of the n-type half-Heusler alloy Hf0. 3Zr0. 7NiSn0. $98 \mathrm{Sb} 0$. 02. Mater. Today Phys. 2019, 9, 100134.

(6) Graf, T.; Felser, C.; Parkin, S. S. Simple rules for the understanding of Heusler compounds. Prog. Solid State Chem. 2011, 39, 1-50.

(7) Anand, S.; Xia, K.; Hegde, V. I.; Aydemir, U.; Kocevski, V.; Zhu, T.; Wolverton, C.; Snyder, G. J. A valence balanced rule for discovery of 18-electron half-Heuslers with defects. Energy Environ. Sci. 2018, 11, 1480-1488.

(8) Bos, J.-W. G.; Downie, R. A. Half-Heusler thermoelectrics: a complex class of materials. J. Phys.: Condens. Matter 2014, 26, 433201.

(9) Snyder, G. J.; Snyder, A. H.; Wood, M.; Gurunathan, R.; Snyder, B. H.; Niu, C. Weighted Mobility. Advanced Materials 2020, 2001537.

(10) Xin, J.; Tang, Y.; Liu, Y.; Zhao, X.; Pan, H.; Zhu, T. Valleytronics in thermoelectric materials. npj Quant. Mater. 2018, 3, 1-10.

(11) Liu, Z.; Guo, S.; Wu, Y.; Mao, J.; Zhu, Q.; Zhu, H.; Pei, Y.; Sui, J.; Zhang, Y.; Ren, Z. Design of high-performance disordered half-Heusler thermoelectric materials using 18-electron rule. Adv. Funct. Mater. 2019, 29, 1905044.

(12) Pei, Y.; Wang, H.; Snyder, G. J. Band engineering of thermoelectric materials. Adv. Mater. 2012, 24, 6125-6135.

(13) Pei, Y.; Shi, X.; LaLonde, A.; Wang, H.; Chen, L.; Snyder, G. J. Convergence of electronic bands for high performance bulk thermoelectrics. Nature 2011, 473, 66-69.

(14) Fu, C.; Bai, S.; Liu, Y.; Tang, Y.; Chen, L.; Zhao, X.; Zhu, T. Realizing high figure of merit in heavy-band p-type half-Heusler thermoelectric materials. Nat. commun. 2015, 6, 8144 . 
(15) Guo, S.; Jia, T.; Zhang, Y. Electrical Property Dominated Promising Half-Heusler Thermoelectrics through High-Throughput Material Computations. J. Phys. Chem. C 2019, 123, 18824-18833.

(16) Feng, Z.; Fu, Y.; Putatunda, A.; Zhang, Y.; Singh, D. J. Electronic structure as a guide in screening for potential thermoelectrics: Demonstration for half-Heusler compounds. Phys. Rev. B 2019, 100, 085202.

(17) Zhu, H. et al. Discovery of TaFeSb-based half-Heuslers with high thermoelectric performance. Nat. commun. 2019, 10, 270.

(18) Zhu, H.; He, R.; Mao, J.; Zhu, Q.; Li, C.; Sun, J.; Ren, W.; Wang, Y.; Liu, Z.; Tang, Z., et al. Discovery of $\mathrm{ZrCoBi}$ based half Heuslers with high thermoelectric conversion efficiency. Nat. Commun. 2018, 9, 1-9.

(19) Sakurada, S.; Shutoh, N. Effect of Ti substitution on the thermoelectric properties of (Zr, Hf) NiSn half-Heusler compounds. Appl. Phys. Lett. 2005, 86, 082105.

(20) Gong, B.; Li, Y.; Liu, F.; Zhu, J.; Wang, X.; Ao, W.; Zhang, C.; Li, J.; Xie, H.; Zhu, T. Continuously enhanced structural disorder to suppress the lattice thermal conductivity of ZrNiSn-based half-Heusler alloys by multielement and multisite alloying with very low Hf content. ACS Appl. Mater. Interfaces 2019, 11, 13397-13404.

(21) Chauhan, N. S.; Raghuvanshi, P. R.; Tyagi, K.; Johari, K. K.; Tyagi, L.; Gahtori, B.; Bathula, S.; Bhattacharya, A.; Mahanti, S. D.; Singh, V. N., et al. Defect Engineering for Enhancement of Thermoelectric Performance of (Zr, Hf) NiSn-Based n-type HalfHeusler Alloys. J. Phys. Chem. C 2020, 124, 8584-8593.

(22) Zeier, W. G.; Schmitt, J.; Hautier, G.; Aydemir, U.; Gibbs, Z. M.; Felser, C.; Snyder, G. J. Engineering half-Heusler thermoelectric materials using Zintl chemistry. Nat. Rev. Mater. 2016, 1, 16032. 
(23) Dylla, M. T.; Dunn, A.; Anand, S.; Jain, A.; Snyder, G. J. Machine learning chemical guidelines for engineering electronic structures in half-heusler thermoelectric materials. Research 2020, 2020 .

(24) Kresse, G.; Joubert, D. From ultrasoft pseudopotentials to the projector augmentedwave method. Phys. Rev. B 1999, 59, 1758.

(25) Perdew, J. P.; Burke, K.; Ernzerhof, M. Generalized gradient approximation made simple. Phys. Rev. Lett. 1996, r7, 3865.

(26) Monkhorst, H. J.; Pack, J. D. Special points for Brillouin-zone integrations. Phys. Rev. $B$ 1976, 13, 5188 .

(27) Madsen, G. K.; Carrete, J.; Verstraete, M. J. BoltzTraP2, a program for interpolating band structures and calculating semi-classical transport coefficients. Comput. Phys. Commun. 2018, 231, 140-145.

(28) Gibbs, Z. M.; Ricci, F.; Li, G.; Zhu, H.; Persson, K.; Ceder, G.; Hautier, G.; Jain, A.; Snyder, G. J. Effective mass and Fermi surface complexity factor from ab initio band structure calculations. npj Comput. Mater. 2017, 3, 1-7.

(29) Wang, H.; LaLonde, A. D.; Pei, Y.; Snyder, G. J. The criteria for beneficial disorder in thermoelectric solid solutions. Adv. Funct. Mater. 2013, 23, 1586-1596.

(30) Zunger, A.; Wei, S.-H.; Ferreira, L.; Bernard, J. E. Special quasirandom structures. Phys. Rev. Lett. 1990, 65, 353.

(31) Hao, S.; Zhao, L.-D.; Chen, C.-Q.; Dravid, V. P.; Kanatzidis, M. G.; Wolverton, C. M. Theoretical prediction and experimental confirmation of unusual ternary ordered semiconductor compounds in $\mathrm{Sr}-\mathrm{Pb}-\mathrm{S}$ system. Journal of the American Chemical Society 2014, 136, 1628-1635. 
(32) Jiang, C.; Wolverton, C.; Sofo, J.; Chen, L.-Q.; Liu, Z.-K. First-principles study of binary bcc alloys using special quasirandom structures. Phys. Rev. B 2004, 69, 214202.

(33) Guo, S.; Liu, Z.; Feng, Z.; Jia, T.; Anand, S.; Snyder, G. J.; Zhang, Y. Prediction of improved thermoelectric performance by ordering in double half-Heusler materials. $J$. Mater. Chem. A 2020, 8, 23590-23598.

(34) Wolverton, C. Crystal structure and stability of complex precipitate phases in $\mathrm{Al}-\mathrm{Cu}^{-}$ $\mathrm{Mg}-(\mathrm{Si})$ and $\mathrm{Al}-\mathrm{Zn}-\mathrm{Mg}$ alloys. Acta Mater. 2001, 49, 3129-3142.

(35) Medeiros, P. V.; Stafström, S.; Björk, J. Effects of extrinsic and intrinsic perturbations on the electronic structure of graphene: Retaining an effective primitive cell band structure by band unfolding. Phys. Rev. B 2014, 89, 041407.

(36) Zhang, X.; Bu, Z.; Shi, X.; Chen, Z.; Lin, S.; Shan, B.; Wood, M.; Snyder, A. H.; Chen, L.; Snyder, G. J., et al. Electronic quality factor for thermoelectrics. Sci. Adv. 2020, 6, eabc0726.

(37) Park, J.; Dylla, M.; Xia, Y.; Wood, M.; Snyder, G. J.; Jain, A. When band convergence is not beneficial for thermoelectrics. Nat. Commun. 2021, 12, 1-8.

(38) Dylla, M. T.; Kang, S. D.; Snyder, G. J. Effect of Two-Dimensional Crystal Orbitals on Fermi Surfaces and Electron Transport in Three-Dimensional Perovskite Oxides. Angew. Chemie 2019, 131, 5557-5566.

(39) Brod, M. K.; Snyder, G. J. Orbital chemistry of high valence band convergence and low-dimensional topology in PbTe. J. Mater. Chem. A 2021, 9, 12119-12139.

(40) Slater, J. C.; Koster, G. F. Simplified LCAO method for the periodic potential problem. Phys. Rev. 1954, 94, 1498.

(41) Harrison, W. A. Electronic structure and the properties of solids: the physics of the chemical bond; Courier Corporation, 2012. 
(42) Zeier, W. G.; Zevalkink, A.; Gibbs, Z. M.; Hautier, G.; Kanatzidis, M. G.; Snyder, G. J. Thinking like a chemist: intuition in thermoelectric materials. Angew. Chem. Int. Ed 2016, 55, 6826-6841.

(43) Rohrer, G. S. Structure and bonding in crystalline materials; Cambridge University Press, 2001. 


\section{TOC Graphic}

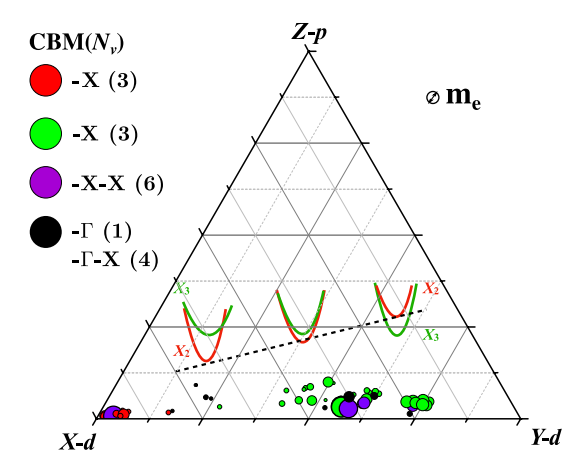

